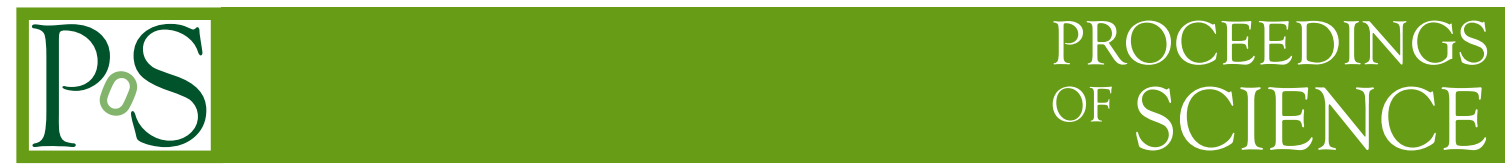

\title{
Review on Flavianet analyses
}

\author{
Matteo Palutan*广 \\ Laboratori Nazionali di Frascati dell'INFN, Frascati, Italy \\ E-mail: Matteo.Palutan@lnf.infn.it
}

We present a global analysis of leptonic and semileptonic kaon decay data, including all recent results published by the BNL-E865, KLOE, KTeV, ISTRA+ and NA48 experiments. This analysis, in conjunction with precise lattice calculations of the hadronic matrix elements now available, leads to a very accurate determination of $V_{\text {us }}$, and allows us to perform several stringent tests of the Standard Model.

2009 KAON International Conference KAON09,

June 09 - 122009

Tsukuba, Japan

\footnotetext{
*Speaker.

$\dagger$ on behalf of the Flavianet Working Group on Kaon decays.
} 


\section{Introduction}

At present, the first-row constraint $\left|V_{\mathrm{ud}}\right|^{2}+\left|V_{\mathrm{us}}\right|^{2}+\left|V_{\mathrm{ub}}\right|^{2}=1$ (with $\left|V_{\mathrm{ub}}\right|^{2}$ negligible) offers the most precise test of CKM unitarity. An experimental deviation from that prediction would be evidence for "new physics" beyond Standard Model (SM) expectations in the form of tree or loop contributions to muon decay and/or the semileptonic processes from which the $V_{i j}$ values are extracted. Up until 2002 (and for the 2004 PDG evaluation [1]), the evaluation of $V_{\text {us }}$ from older $K \rightarrow \pi l v\left(K_{l 3}\right)$ data gave $2.3 \sigma$ hint of unitarity violation in the first-row test. The 2003 measurement of $\mathrm{BR}\left(K_{e 3}^{+}\right)$by BNL E865 [2] gave a value for $\left|V_{\mathrm{us}}\right|$ consistent with unitarity. In the period 2004-2006, many new measurements of kaon branching ratios (BR), lifetimes $(\tau)$, and form-factor slopes $(\lambda)$ were announced by KLOE, KTeV, ISTRA+ and NA48. All of these new measurements are distinguished from the older ones in that they are based on much higher statistics, and in that radiative corrections are applied consistently. I present here an up-to-date evaluation of $V_{\text {us }}$ from the combination of leptonic pion and kaon decays and from semileptonic kaon decays. The combination of experimental results has been carried out by the FlaviaNet Working Group on Kaon decays, and has been first reported in Ref. [3].

$V_{\text {us }}$ is related to the kaon semileptonic decay rate through the following equation:

$$
\Gamma\left(K_{l 3}\right)=\frac{C_{K}^{2} G_{F}^{2} M_{K}^{5}}{192 \pi^{3}} S_{E W}\left|V_{\mathrm{us}}\right|^{2}\left|f_{+}(0)\right|^{2} I_{K l}(\lambda)\left(1+2 \Delta_{K}^{S U(2)}+2 \Delta_{K l}^{E M}\right),
$$

where $K=K^{0}, K^{ \pm}, l=e, \mu$ and $C_{K}$ is a Clebsch-Gordan coefficient, equal to $1 / 2$ and 1 for $K^{ \pm}$and $K^{0}$, respectively. In the above expression, the decay width $\Gamma\left(K_{l 3}\right)$ is experimentally determined by measuring the kaon lifetime and the semileptonic BRs. A well determined treatment of the radiative decays is required in order to use the measured BR in the expression 1.1; usually, most recent experiments publish values of BR totally inclusive of radiation. The hadronic matrix element (form factor) for the $K \rightarrow \pi$ transition is parameterized in terms of its value at zero momentum transfer for neutral kaon decays, $f_{+}(0) \equiv f_{+}^{K^{0} \pi^{-}}(0)$, which is determined from theory. Form factor dependence on the momentum is described by one or more slope parameters $\lambda$, which are measured from the decay spectra, and is integrated over the decay phase space, giving rise to the $I_{K l}(\lambda)$ integral in equation 1.1. On top of that, some higher order corrections have to be computed from theory: $S_{E W}=1.0232$ is the universal short-distance electroweak correction; $\Delta_{K}^{S U(2)}$ and $\Delta_{K l}^{E M}$ are $S U(2)$-breaking and long-distance electromagnetic corrections, which depend on the kaon charge and on the lepton flavor.

In the following section 2 the most recent measurements for $K_{L}, K_{S}$ and $K^{ \pm}$BRs and lifetimes will be reviewed and the updated averages will be presented; in section 3 the measurement of the form factor slopes and the evaluation of the phase space integrals will be summarized; in sections $₫$ and 5 the extraction on $V_{\text {us }}$ will be finally addressed.

\section{Combination of experimental data}

We perform fits to world data on the BRs and lifetimes for the $K_{L}, K_{S}$, and $K^{ \pm}$, with the constraint that BRs add to unity. A detailed description of the fit is given in Ref. [3]. The present version of our fits uses only published measurements. 


\section{1 $K_{L}$ leading branching ratios and lifetime}

Many new measurements of $K_{L}$ BRs were performed during the last years, which contributed to clarify the $K_{L}$ experimental picture with respect to the 2004 PDG compilation [ $₫$ ]. The KTeV experiment has measured accurately five ratios of $K_{L}$ main decay widths from independent samples of $10^{5}-10^{6}$ events collected with a single trigger. They obtain [§] : $\Gamma\left(K_{\mu 3}\right) / \Gamma\left(K_{e 3}\right)=0.6640(26)$, $\Gamma\left(\pi^{+} \pi^{-} \pi^{0}\right) / \Gamma\left(K_{e 3}\right)=0.3078(18), \Gamma\left(\pi^{+} \pi^{-}\right) / \Gamma\left(K_{e 3}\right)=0.004856(28), \Gamma\left(2 \pi^{0}\right) / \Gamma\left(3 \pi^{0}\right)=0.004446$ (25), and $\Gamma\left(3 \pi^{0}\right) / \Gamma\left(K_{e 3}\right)=0.4782(55)$. The measured ratios are used in the global fit to $K_{L}$ BRs and lifetime, with correlations provided by the experiment. The NA48 experiment has measured the ratio of $K_{e 3}$ decays normalized to final states with two charged tracks, obtained from a sample of $80 \times 10^{6}$ events. They find [6] $\Gamma\left(K_{e 3}\right) / \Gamma(2$ track $)=0.4978(35)$. The measured ratio is used in the present fit to $K_{L}$ decay modes. The KLOE experiment has measured the absolute BRs for the four main $K_{L}$ decay channels from a sample of $13 \times 10^{6} \phi \rightarrow K_{S} K_{L}$ events with a $K_{S} \rightarrow \pi^{+} \pi^{-}$decay reconstructed in the apparatus. The results depend on the $K_{L}$ lifetime through the geometrical acceptance of the apparatus as $d \mathrm{BR} / \mathrm{BR}=0.67 d \tau_{L} / \tau_{L}$. Using as reference value $\tau_{L}^{(0)}=51.54 \mathrm{~ns}$ they get [7] $\mathrm{BR}^{(0)}\left(K_{e 3}\right)=0.4049(21), \mathrm{BR}^{(0)}\left(K_{\mu 3}\right)=0.2726(16), \mathrm{BR}^{(0)}\left(3 \pi^{0}\right)=0.2018(24)$, and $\mathrm{BR}^{(0)}\left(\pi^{+} \pi^{-} \pi^{0}\right)=0.1276(15)$. For the global $K_{L}$ fit, these values have been used accounting for lifetime dependence and other experimental correlations. KLOE has provided also an independent measurement of $\tau_{L}$, obtained by fitting the proper decay time distribution for $K_{L} \rightarrow 3 \pi^{0}$ events, for which the reconstruction efficiency is high and uniform over a fiducial volume of $\sim 0.4 \lambda_{L}$. They find [8] $\tau_{L}=50.92(30) \mathrm{ns}$.

All of the results discussed above, plus few more which are described in Ref. [3], are used in a PDG-like fit to evaluate the $K_{L}$ main decay channels and lifetime. The only constraint used in this fit is $\Sigma(\mathrm{BR})=1$. The fit converges succesfully with $\chi^{2} / n d f=19.8 / 12$ (Prob $=7.1 \%$ ), and the results are reported in Table 1. Our definition of $\mathrm{BR}\left(\pi^{+} \pi^{-}\right)$is fully inclusive of inner bremsstrahlung (IB), but exclusive of the direct emission (DE) component; radiative modes are given separately, for $E_{\gamma}^{*}>20 \mathrm{MeV}$.

Table 1: $K_{L}$ BRs and lifetime from a fit to recent data.

\begin{tabular}{ccc}
\hline \hline Parameter & Value & Scale factor \\
\hline $\operatorname{BR}\left(K_{e 3}\right)$ & $0.4056(9)$ & 1.3 \\
$\operatorname{BR}\left(K_{\mu 3}\right)$ & $0.2704(10)$ & 1.5 \\
$\operatorname{BR}\left(3 \pi^{0}\right)$ & $0.1952(9)$ & 1.2 \\
$\operatorname{BR}\left(\pi^{+} \pi^{-} \pi^{0}\right)$ & $0.1254(6)$ & 1.1 \\
$\operatorname{BR}\left(\pi^{+} \pi^{-}\right)$ & $1.967(7) \times 10^{-3}$ & 1.1 \\
$\operatorname{BR}\left(\pi^{+} \pi^{-} \gamma\right)$ & $4.15(9) \times 10^{-5}$ & 1.6 \\
$\operatorname{BR}\left(\pi^{+} \pi^{-} \gamma_{\text {DE }}\right)$ & $2.84(8) \times 10^{-5}$ & 1.3 \\
$\operatorname{BR}\left(2 \pi^{0}\right)$ & $8.65(9) \times 10^{-4}$ & 1.4 \\
$\operatorname{BR}(\gamma \gamma)$ & $5.47(4) \times 10^{-4}$ & 1.1 \\
$\tau_{L}$ & $51.16(21) \mathrm{ns}$ & 1.1 \\
\hline
\end{tabular}

Figure 11 shows a comparison between previous PDG averages and the present results for $K_{e 3}$, $K_{\mu 3}, 3 \pi^{0}$ and $\pi^{+} \pi^{-}$decay channels. Differences between this fit and 2008 PDG edition [9] are 
minor, while a substantial difference is observed with respect to the 2004 PDG review [4], due to a completely renewed set of measurements.

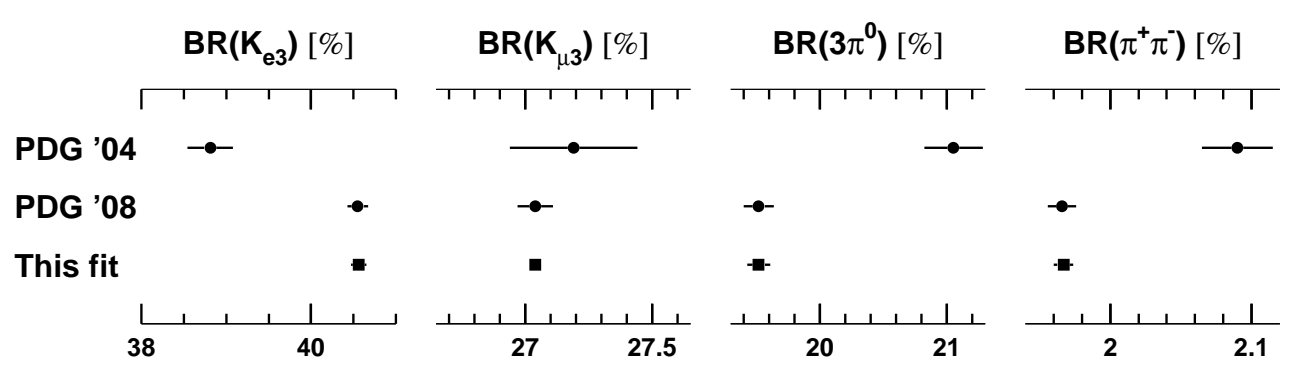

Figure 1: BR evolution for some representative $K_{L}$ channels: $K_{e 3}, K_{\mu 3}, 3 \pi^{0}$, and $\pi^{+} \pi^{-}$.

\section{2 $K_{S}$ leading branching ratios and lifetime}

$\mathrm{KLOE}$ has measured the ratio $\mathrm{BR}\left(K_{S} \rightarrow \pi e v\right) / \mathrm{BR}\left(K_{S} \rightarrow \pi^{+} \pi^{-}\right)$with $1.3 \%$ precision [10], making possible an independent determination of $\left|V_{u s}\right| f_{+}(0)$ to better than $0.7 \%$. In Ref. [11], KLOE combines the above measurement with their measurement $\operatorname{BR}\left(K_{S} \rightarrow \pi^{+} \pi^{-}\right) / \operatorname{BR}\left(K_{S} \rightarrow\right.$ $\left.\pi^{0} \pi^{0}\right)=2.2459(54)$. Using the constraint that the $K_{S}$ BRs sum to unity and assuming the universality of lepton couplings, they determine the BRs for $\pi^{+} \pi^{-}, \pi^{0} \pi^{0}, K_{e 3}$, and $K_{\mu 3}$ decays. Our fit is an extension of the analysis in [11], which makes also use of a recent determination of $\Gamma\left(K_{S} \rightarrow \pi e v\right) / \Gamma\left(K_{L} \rightarrow \pi e v\right)$ [12] by NA48, where the denominator is obtained from the results of our $K_{L}$ fit. As far as the $K_{S}$ lifetime is concerned, we include in the fit the measurements by NA48 [13] and KTeV [14], performed without assumption of CPT symmetry. The results of the fit are listed in Table 2 .

Table 2: Results of fit to $K_{S}$ BRs and lifetime

\begin{tabular}{lcc}
\hline \hline Parameter & Value & Scale factor \\
\hline $\operatorname{BR}\left(\pi^{+} \pi^{-}\right)$ & $0.6920(5)$ & 1.0 \\
$\operatorname{BR}\left(\pi^{0} \pi^{0}\right)$ & $0.3069(5)$ & 1.0 \\
$\operatorname{BR}\left(K_{e 3}\right)$ & $7.05(8) \times 10^{-4}$ & 1.0 \\
$\operatorname{BR}\left(K_{\mu 3}\right)$ & $4.66(6) \times 10^{-4}$ & 1.0 \\
$\tau_{S}$ & $0.08958(5) \mathrm{ns}$ & 1.0 \\
\hline
\end{tabular}

\section{3 $K^{ \pm}$leading branching ratios and lifetime}

There are several new results providing information on $K_{\ell 3}^{ \pm}$rates. The NA48/2 collaboration has published measurements of the $K_{\ell 3}$ decay rates normalized to $\pi \pi^{0}$ final state [15], BR $\left(K_{e 3}\right) / \mathrm{BR}$ $\left(\pi \pi^{0}\right)=0.2470(10)$ and $\operatorname{BR}\left(K_{\mu 3}\right) / \operatorname{BR}\left(\pi \pi^{0}\right)=0.1637(7)$ In our fit we use these values, and take their correlation into account. KLOE has measured the absolute BRs for the $K_{e 3}$ and $K_{\mu 3}$ decays [16]. In $\phi \rightarrow K^{+} K^{-}$events, $K^{+}$decays into $\mu v$ or $\pi \pi^{0}$ are used to tag a $K^{-}$beam, and vice 
versa. KLOE performs four separate measurements for each $K_{\ell 3} \mathrm{BR}$, corresponding to the different combinations of kaon charge and tagging decay. The final averages are $\operatorname{BR}\left(K_{e 3}\right)=4.965(53)(38) \%$ and $\operatorname{BR}\left(K_{\mu 3}\right)=3.233(29)(26) \%$. KLOE has also measured the absolute branching ratio for the $\pi \pi^{0}$ [17] and $\mu \nu$ decay [18]. Our fit takes into account the correlation between these values, as well as their dependence on the $K^{ \pm}$lifetime. The world average value for $\tau_{ \pm}$is nominally quite precise. However, the PDG error is scaled by 2.1 ; the confidence level for the average is $0.17 \%$. It is important to confirm the value of $\tau_{ \pm}$. The new measurement from KLOE [19], $\tau_{ \pm}=12.347(30) \mathrm{ns}$, agrees with the PDG average.

Our fit for the six largest $K^{ \pm}$branching ratios and lifetime includes the the six measurements noted above, together with some older results (as described in Ref. [3]). We have recently carried out a comprehensive survey of the $K^{ \pm}$data set, which led to the elimination of 11 measurements currently in the 2008 PDG fit. Our fit uses 17 input measurements, seven free parameters, and one constraint, giving 11 degrees of freedom. We obtain the results in Table 3 . The fit gives $\chi^{2}=25.8$ $(P=0.69 \%)$. The comparatively low $P$-value reflects some tension between the KLOE and NA48/2 measurements of the $K_{\ell 3}$ branching ratios. Both the significant evolution of the average values of

\begin{tabular}{lcc}
\multicolumn{3}{c}{ Table 3: Results of fit to $K^{ \pm}$BRs and lifetime. } \\
\hline \hline Parameter & Value & Scale factor \\
\hline $\operatorname{BR}\left(K_{\mu 2}\right)$ & $63.47(18) \%$ & 1.3 \\
$\operatorname{BR}\left(\pi \pi^{0}\right)$ & $20.61(8) \%$ & 1.1 \\
$\operatorname{BR}(\pi \pi \pi)$ & $5.573(16) \%$ & 1.2 \\
$\operatorname{BR}\left(K_{e 3}\right)$ & $5.078(31) \%$ & 1.3 \\
$\operatorname{BR}\left(K_{\mu 3}\right)$ & $3.359(32) \%$ & 1.9 \\
$\operatorname{BR}\left(\pi \pi^{0} \pi^{0}\right)$ & $1.757(24) \%$ & 1.0 \\
$\tau_{ \pm}$ & $12.384(15) \mathrm{ns}$ & 1.2 \\
\hline
\end{tabular}

the $K_{\ell 3}$ BRs and the effect of the correlations with $\mathrm{BR}\left(\pi \pi^{0}\right)$ are evident in Fig. 2.

\section{Form factor slopes and determination of $K_{l 3}$ decay phase space}

Since $K \rightarrow \pi$ is a $0^{-} \rightarrow 0^{-}$transition, only the vector part of the weak current has a nonvanishing contribution. The matrix element can be expressed as

$$
\left\langle\pi\left|J_{\mu}^{V}\right| K\right\rangle=\left[(P+p)_{\mu} f_{+}(t)+(P-p)_{\mu} f_{-}(t)\right]
$$

where $P, p$, are the kaon, pion momenta, and $t=(P-p)^{2}$ is the only $L$-invariant variable. The term proportional to $f_{-}(t)$ is only relevant for $K_{\mu 3}$ decays, since it is multiplied by the lepton mass. It is customary to expand the vector form factor $f_{+}(t)$ as

$$
f_{+}(t)=f_{+}(0)\left[1+\lambda_{+}^{\prime} \frac{t}{m^{2}}+\frac{1}{2} \lambda_{+}^{\prime \prime}\left(\frac{t}{m^{2}}\right)^{2}+\ldots\right],
$$

where $m$ is the mass of the charged pion, and only linear and quadratic terms are retained. In the above expression, the form factor at zero momentum transfer, $f_{+}(0)$, is evaluated from theory, while the form factor slopes, $\lambda_{+}^{\prime}, \lambda_{+}^{\prime \prime}$ are experimentally determined from semileptonic decay spectra. A 

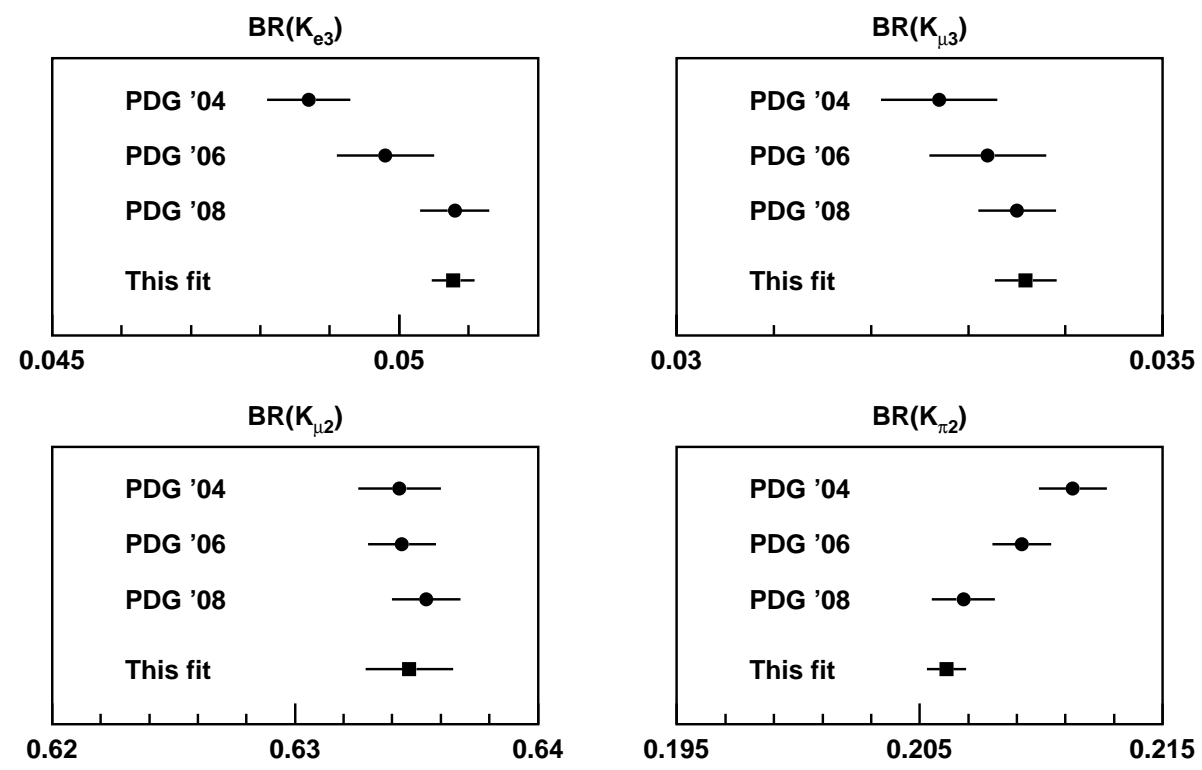

Figure 2: Evolution of average values for main $K^{ \pm}$BRs.

Table 4: Average of quadratic fit results for $K_{e 3}$ slopes.

\begin{tabular}{lcc}
\hline \hline & $\begin{array}{c}K_{L} \text { and } K^{-} \text {data } \\
4 \text { measurements } \\
\chi^{2} / \mathrm{ndf}=5.3 / 6(51 \%)\end{array}$ & $\begin{array}{c}K_{L} \text { data only } \\
3 \text { measurements } \\
\chi^{2} / \mathrm{ndf}=4.7 / 4(32 \%)\end{array}$ \\
\hline$\lambda_{+}^{\prime} \times 10^{3}$ & $25.2 \pm 0.9$ & $24.9 \pm 1.1$ \\
$\lambda_{+}^{\prime \prime} \times 10^{3}$ & $1.6 \pm 0.4$ & $1.6 \pm 0.5$ \\
$\rho\left(\lambda_{+}^{\prime}, \lambda_{+}^{\prime \prime}\right)$ & -0.94 & -0.95 \\
$I\left(K_{e 3}^{0}\right)$ & $0.15463(21)$ & $0.15454(29)$ \\
$I\left(K_{e 3}^{ \pm}\right)$ & $0.15900(22)$ & $0.15890(30)$ \\
\hline
\end{tabular}

scalar form factor $f_{0}(t)$ is introduced in the parametrization of $f_{-}(t)$, defined as $f_{-}(t)=\left(f_{0}(t)-\right.$ $\left.f_{+}(t)\right)\left(M_{K}^{2}-m^{2}\right) / t$ with $f_{0}(0)=f_{+}(0)$. As in the case of the vector form factor, the scalar form factor is expanded in powers of momentum transfer $t$.

For $K_{e 3}$ decays, recent measurements of the quadratic slope parameters of the vector form factor, $\lambda_{+}^{\prime}$ and $\lambda_{+}^{\prime \prime}$, are available from KTeV [20], KLOE [21], ISTRA+ [22], and NA48 [23]. We

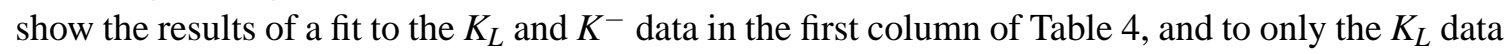
in the second column. With correlations correctly taken into account, both fits give good values of $\chi^{2} /$ ndf. The significance of the quadratic term is $4.2 \sigma$ from the fit to all data, and $3.5 \sigma$ from the fit to $K_{L}$ data only. Including or excluding the $K^{-}$slopes has little impact on the values of $\lambda_{+}^{\prime}$ and $\lambda_{+}^{\prime \prime}$; in particular, the values of the phase-space integrals change by just $0.06 \%$. KLOE, KTeV, and 
Table 5: Pole fit results for $K_{e 3}^{0}$ slopes.

\begin{tabular}{lc|c}
\hline \hline Experiment & $M_{V}(\mathrm{MeV})$ & $\left\langle M_{V}\right\rangle=875 \pm 5 \mathrm{MeV}$ \\
$\mathrm{KLOE}$ & $870 \pm 6 \pm 7$ & $\chi^{2} / \mathrm{ndf}=1.8 / 2$ \\
$\mathrm{KTeV}$ & $881.03 \pm 7.11$ & $\lambda_{+}^{\prime} \times 10^{3}=25.42(31)$ \\
$\mathrm{NA} 48$ & $859 \pm 18$ & $\lambda_{+}^{\prime \prime}=2 \times \lambda_{+}^{\prime 2}$ \\
& & $I\left(K_{e 3}^{0}\right)=0.15470(19)$ \\
\hline
\end{tabular}

NA48 also used a pole parameterization for the vector form factor,

$$
f_{+}(t)=f_{+}(0) \frac{M_{V}^{2}}{M_{V}^{2}-t},
$$

in which dominance of a single resonance is assumed and its mass $M_{V}$ is the fit parameter. Results for $M_{V}$ from pole fits to $K_{L e 3}$ data are quoted in Table 5 . The average value of $M_{V}$ from all three experiments is $M_{V}=875 \pm 5 \mathrm{MeV}$ with $\chi^{2} / \mathrm{ndf}=1.8 / 2$. The three values are quite compatible with each other and reasonably close to the known value of the $K^{ \pm *}(892)$ mass $(891.66 \pm 0.26 \mathrm{MeV})$. The values for $\lambda_{+}^{\prime}$ and $\lambda_{+}^{\prime \prime}$ from expansion of the pole parametrization are qualitatively in agreement with the average of the quadratic fit results. More importantly, for the evaluation of the phase-space integrals, using the average of quadratic or pole fit results gives values of $I\left(K_{e 3}^{0}\right)$ that differ by just $0.05 \%$.

For $K_{\mu 3}$ decays, recent measurements of the slope parameters $\lambda_{+}^{\prime}, \lambda_{+}^{\prime \prime}$, and $\lambda_{0}$ are available from $\mathrm{KTeV}$ [20], KLOE [24], ISTRA+ [25], and NA48 [26]. We will not use the ISTRA+ result for the average because systematic errors have not been provided. We use the $K_{e 3}-K_{\mu 3}$ averages provided by the experiments for $\mathrm{KTeV}$ and KLOE. NA48 does not provide such an average, so we calculate it for inclusion in the fit. We have studied the statistical sensitivity of the form-factor slope measurements using Monte Carlo techniques. The conclusions of this study are a) that neglecting a quadratic term in the parameterization of the scalar form factor when fitting results leads to a shift of the value of the linear term by about 3.5 times the value of the quadratic term; and b) that because of correlations, it is impossible to measure the quadratic slope parameter from quadratic fits to the data at any plausible level of statistics. The use of the linear representation of the scalar form factor is thus inherently unsatisfactory, but has little impact in the extraction of $V_{\text {us }}$. The results of the combination of $K_{\ell 3}$ results are listed in Table 6, and shown in Fig. 3. The value of $\chi^{2} /$ ndf for all measurements is terrible; we quote the results with scaled errors. This leads to errors on the phase-space integrals that are $\sim 60 \%$ larger after inclusion of the $K_{\mu 3}$ NA48 data. The evaluations of the phase-space integrals for all four modes are listed in each case. Correlations are fully accounted for, both in the fits and in the evaluation of the integrals. Adding the $K_{\mu 3}$ data to the fit does not cause drastic changes to the values of the phase-space integrals for the $K_{e 3}$ modes: the values for $I\left(K_{e 3}^{0}\right)$ and $I\left(K_{e 3}^{ \pm}\right)$in Table 6 are qualitatively in agreement with those in Table $\bigoplus$.

\section{Extraction of $\left|V_{\mathrm{us}}\right| f_{+}(0)$}

$S U(2)$-breaking and $E M$ corrections which are used to extract $\left|V_{\mathrm{us}}\right| f_{+}(0)$ are summarized in table 7. The $S U(2)$-breaking correction is evaluated with ChPT to $O\left(p^{4}\right)$, as described in [27], and 
Table 6: Averages of quadratic fit results for $K_{e 3}$ and $K_{\mu 3}$ slopes.

\begin{tabular}{lc}
\hline \hline$\chi^{2} /$ ndf & $29 / 8\left(3 \times 10^{-4}\right)$ \\
$\lambda_{+}^{\prime} \times 10^{3}$ & $24.5 \pm 0.9(S=1.1)$ \\
$\lambda_{+}^{\prime \prime} \times 10^{3}$ & $1.8 \pm 0.4(S=1.3)$ \\
$\lambda_{0} \times 10^{3}$ & $11.7 \pm 1.4(S=1.9)$ \\
$\rho\left(\lambda_{+}^{\prime}, \lambda_{+}^{\prime \prime}\right)$ & -0.94 \\
$\rho\left(\lambda_{+}^{\prime}, \lambda_{0}\right)$ & +0.44 \\
$\rho\left(\lambda_{+}^{\prime \prime}, \lambda_{0}\right)$ & -0.52 \\
$I\left(K_{e 3}^{0}\right)$ & $0.15449(20)$ \\
$I\left(K_{e 3}^{ \pm}\right)$ & $0.15885(21)$ \\
$I\left(K_{\mu 3}^{0}\right)$ & $0.10171(32)$ \\
$I\left(K_{\mu 3}^{ \pm}\right)$ & $0.10467(33)$ \\
$\rho\left(I_{e 3}, I_{\mu 3}\right)$ & +0.53 \\
\hline
\end{tabular}
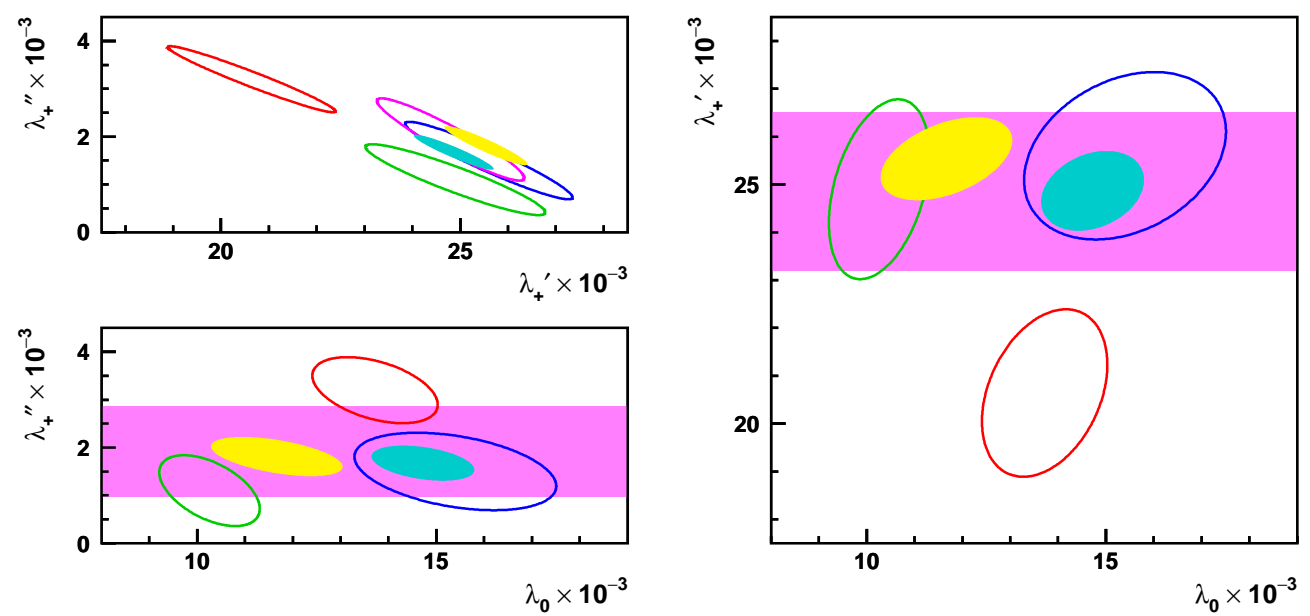

Figure 3: $1-\sigma$ contours for $\lambda_{+}^{\prime}, \lambda_{+}^{\prime \prime}$, and $\lambda_{0}$ determinations from KLOE(blue ellipse), $\mathrm{KTeV}$ (red ellipse), NA48 (green ellipse), and world average with (filled yellow ellipse) and without(filled cyan ellipse) the NA48 $K_{\mu 3}$ result.

using a recent evaluation of quark mass ratio from [30]. The long distance $E M$ corrections to the full inclusive decay rate are evaluated with ChPT to $O\left(e^{2} p^{2}\right)$ [28], and using low-energy constants from Refs. [29] [30]. The quoted errors are estimates of the only partially known higher order contributions. Using all of the experimental and theoretical inputs discussed above, the values of $\left|V_{\text {us }}\right| f_{+}(0)$ have been evaluated for $K_{L} e 3, K_{L} \mu 3, K_{S} e 3, K^{ \pm} e 3$, and $K^{ \pm} \mu 3$ decay modes, as shown in table 8 and figure 4 . The five decay modes agree well within the quoted errors, and average to

$$
\left|V_{\mathrm{us}}\right| f_{+}(0)=0.2166(5),
$$

with fit probability 55\%. To evaluate the reliability of the $S U(2)$-breaking correction, a comparison is made between separate averages of $\left|V_{\mathrm{us}}\right| f_{+}(0)$ for the neutral and the charged channels, from 
Table 7: Summary of $S U(2)$-breaking and $E M$ corrections.

\begin{tabular}{ccc}
\hline \hline Mode & $\Delta_{K}^{S U(2)}$ & $\Delta_{K l}^{E M}$ \\
\hline$K^{0} e 3$ & 0 & $0.50(11) \%$ \\
$K^{0} \mu 3$ & 0 & $0.70(11) \%$ \\
$K^{ \pm} e 3$ & $2.9(4) \%$ & $0.05(13) \%$ \\
$K^{ \pm} \mu 3$ & $2.9(4) \%$ & $-0.01(13) \%$ \\
\hline
\end{tabular}

Table 8: Values of $\left|V_{\text {us }}\right| f_{+}(0)$ extracted from $K_{l 3}$ decay rates; all sources contributing to the total fractional error are reported separately.

\begin{tabular}{ccc|cccc}
\hline \hline & & & \multicolumn{4}{|c}{ Approx. contrib. to \% err } \\
Mode & $\left|V_{\text {us }}\right| f_{+}(0)$ & $\%$ err & BR & $\tau$ & $\Delta$ & $I_{K l}(\lambda)$ \\
\hline$K_{L} e 3$ & $0.2165(5)$ & 0.26 & 0.09 & 0.20 & 0.11 & 0.06 \\
$K_{L} \mu 3$ & $0.2175(6)$ & 0.32 & 0.15 & 0.18 & 0.15 & 0.16 \\
$K_{S} e 3$ & $0.2157(13)$ & 0.61 & 0.60 & 0.03 & 0.11 & 0.06 \\
$K^{ \pm} e 3$ & $0.2162(11)$ & 0.52 & 0.31 & 0.09 & 0.41 & 0.06 \\
$K^{ \pm} \mu 3$ & $0.2168(14)$ & 0.65 & 0.47 & 0.08 & 0.42 & 0.16 \\
\hline
\end{tabular}

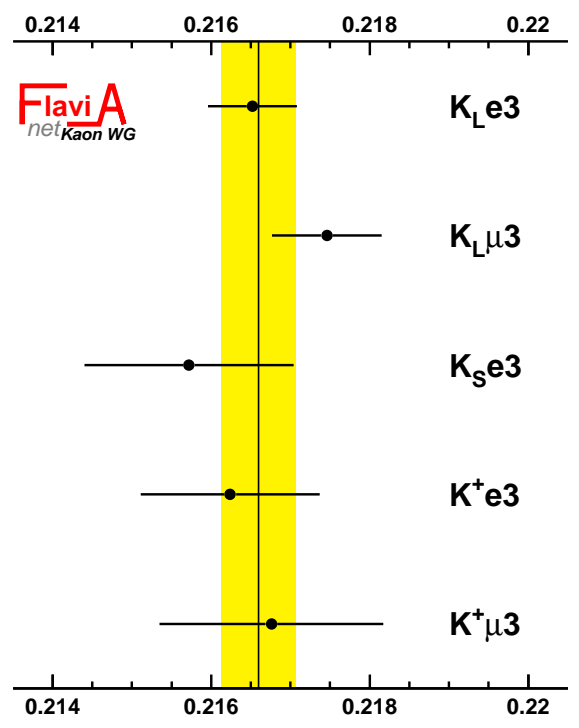

Figure 4: Values of $\left|V_{\mathrm{us}}\right| f_{+}(0)$ extracted from $K_{l 3}$ decay rates; the average between decay modes is indicated by a continuous line.

which we can estimate $\Delta_{\text {exp }}^{S U(2)}=2.7(4) \%$, which is good agreement with the value estimated from theory. 


\section{Determination of $V_{\mathrm{us}}$ and CKM unitarity test}

In the previous section a determination of $\left|V_{\mathrm{us}}\right| f_{+}(0)$ from $K_{l 3}$ decays has been presented, with $\sim 2 \times 10^{-3}$ fractional accuracy. To extract the value of $V_{\text {us }}$, we use the current best determination of $f_{+}(0)$ from lattice QCD,$f_{+}(0)=0.964 \pm 0.005$, from a $2+1$ flavor calculation by the $\mathrm{RBC}+\mathrm{UKQCD}$ collaboration [32]. We get

$$
V_{\mathrm{us}}=0.2246 \pm 0.0012 \text {. }
$$

A value of $V_{\mathrm{us}}$ can also be obtained from a comparison of the radiative inclusive decay rates of $K^{ \pm} \rightarrow \mu^{ \pm} v(\gamma)$ and $\pi^{ \pm} \rightarrow \mu^{ \pm} v(\gamma)$ combined with a lattice calculation of $f_{K} / f_{\pi}$ via [31]

$$
\frac{\Gamma\left(K^{ \pm} \rightarrow \mu^{ \pm} v(\gamma)\right)}{\Gamma\left(\pi^{ \pm} \rightarrow \mu^{ \pm} v(\gamma)\right)}=\frac{\left|V_{\mathrm{us}}\right|^{2} f_{K}^{2} m_{K}\left(1-m_{\mu}^{2} / m_{K}^{2}\right)^{2}}{\left|V_{\mathrm{ud}}\right|^{2} f_{\pi}^{2} m_{\pi}\left(1-m_{\mu}^{2} / m_{\pi}^{2}\right)^{2}} \times 0.9930(35),
$$

with the multiplicative factor coming from the electroweak radiative corrections. To solve equation 5.2 for $V_{\mathrm{us}} / V_{\mathrm{ud}}$, we use the value of $\operatorname{BR}\left(K^{ \pm} \rightarrow \mu^{ \pm} v\right)$ quoted in Sec. 2.3, which is dominated by a recent measurement by KLOE [18] with $0.3 \%$ accuracy, and the lattice result $f_{K} / f_{\pi}=$ $1.189 \pm 0.007$, from HPQCD [33]. From the above results we get

$$
V_{\mathrm{us}} / V_{\mathrm{ud}}=0.2319 \pm 0.0015
$$

This ratio can be used in a fit together with the measurements of $V_{\text {us }}$ from $K_{\ell 3}$ and $V_{\text {ud }}$ from $0^{+} \rightarrow 0^{+}$ nuclear beta decays, $V_{\mathrm{ud}}=0.97425 \pm 0.00022$ [34]. The global fit gives

$$
V_{\mathrm{ud}}=0.97425(22) \quad V_{\mathrm{us}}=0.2252(9) \quad\left[K_{\ell 3, \ell 2}+0^{+} \rightarrow 0^{+}\right],
$$

with $\chi^{2} /$ ndf $=0.52 / 1(47 \%)$. This result does not make use of CKM unitarity. If the unitarity constraint is included, the fit gives

$$
V_{\mathrm{us}}=\sin \theta_{C}=\lambda=0.2253(6) \quad \text { [with unitarity] }
$$

Both results are illustrated in Fig. 5. Using the (rather negligible) $\left|V_{u b}\right|^{2} \simeq 1.5 \times 10^{-5}$ in conjunction with the above results (Eq. 5.4) leads to

$$
\left|V_{u d}\right|^{2}+\left|V_{u s}\right|^{2}+\left|V_{u b}\right|^{2}=0.9999(4)_{V_{u d}}(4)_{V_{u s}}=0.9999(6) \text {. }
$$

The outstanding agreement with unitarity provides an impressive confirmation of Standard Model radiative corrections [35][36] (at about the 60 sigma level!). It can be used to constrain "new physics" effects which, if present, would manifest themselves as a deviation from one, i.e. what would appear to be a breakdown of unitarity (see Ref. [3]).

\section{Charged Higgs Bosons}

A particularly interesting test is the comparison of the $\left|V_{u s}\right|$ value extracted from the helicitysuppressed $K_{\ell 2}$ decays with respect to the value extracted from the helicity-allowed $K_{\ell 3}$ modes. 


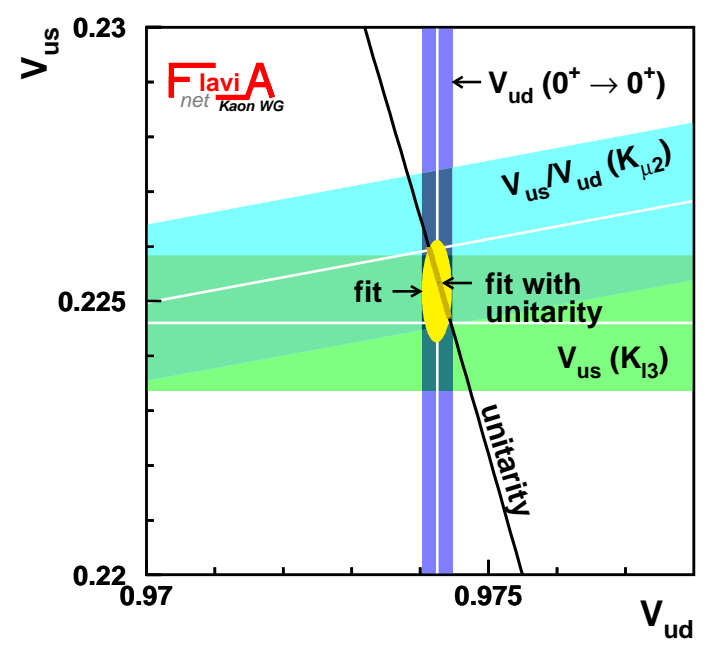

Figure 5: Results of fits to $V_{\mathrm{ud}}, V_{\mathrm{us}}$, and $V_{\mathrm{us}} / V_{\mathrm{ud}}$.

To reduce theoretical uncertainties from $f_{K}$ and electromagnetic corrections in $K_{\ell 2}$, we exploit the ratio $\mathrm{BR}\left(K_{\ell 2}\right) / \mathrm{BR}\left(\pi_{\ell 2}\right)$ and we study the quantity

$$
R_{l 23}=\left|\frac{V_{u s}\left(K_{\ell 2}\right)}{V_{u s}\left(K_{\ell 3}\right)} \times \frac{V_{u d}\left(0^{+} \rightarrow 0^{+}\right)}{V_{u d}\left(\pi_{\ell 2}\right)}\right| .
$$

Within the SM, $R_{l 23}=1$, while deviation from 1 can be induced by non-vanishing scalar- or righthanded currents. Notice that in $R_{l 23}$ the hadronic uncertainties enter through $\left(f_{K} / f_{\pi}\right) / f_{+}(0)$. Effects of scalar currents due to a charged Higgs give [3]

$$
R_{l 23}=\left|1-\frac{m_{K^{+}}^{2}}{M_{H^{+}}^{2}}\left(1-\frac{m_{d}}{m_{s}}\right) \frac{\tan ^{2} \beta}{1+\varepsilon_{0} \tan \beta}\right| \text {. }
$$

In this case, the unitarity relation between $\left|V_{u d}\right|$ extracted from $0^{+} \rightarrow 0^{+}$nuclear beta decays and $\left|V_{u s}\right|$ extracted from $K_{\ell 3}$ remains valid as soon as form factors are experimentally determined. This constraint can be used in the global fit to improve the accuracy of the determination of $R_{l 23}$, which in this scenario turns to be

$$
\left.R_{l 23}\right|_{\mathrm{scalar}} ^{\exp }=1.004 \pm 0.007 .
$$

Here $\left(f_{K} / f_{\pi}\right) / f_{+}(0)$ has been fixed from lattice. This ratio is the key quantity to be improved in order to reduce present uncertainty on $R_{l 23}$. The measurement of $R_{l 23}$ above can be used to set bounds on the charged Higgs mass and $\tan \beta$. Fig. 6 shows the excluded region at $95 \% \mathrm{CL}$ in the $M_{H}-\tan \beta$ plane (setting $\left.\varepsilon_{0}=0.01\right)$. The measurement of $\mathrm{BR}(B \rightarrow \tau \nu)$ [37] can be also used to set a similar bound in the $M_{H}-\tan \beta$ plane. While $B \rightarrow \tau \nu$ can exclude quite an extensive region of this plane, there is an uncovered region in the exclusion corresponding to a destructive interference between the charged-Higgs and the SM amplitude. This region is fully covered by the $K \rightarrow \mu \nu$ result. 


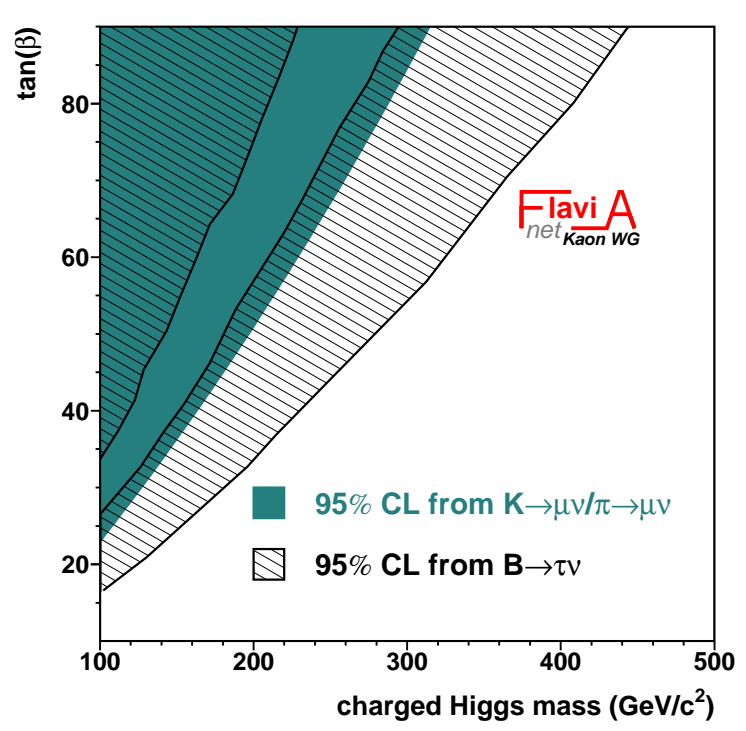

Figure 6: Excluded region in the charged Higgs mass-tan $\beta$ plane. The region excluded by $B \rightarrow \tau \nu$ is also indicated.

\section{References}

[1] F. Gilman, K. Kleinknecht, and B. Renk, in Review of Particle Physics, Phys. Lett. B 592 (2004) 130.

[2] A. Sher at al., Phys. Rev. Lett. 91 (2003) 261802.

[3] M. Antonelli et al., arXiv:0801.1817.

[4] S. Eidelman et al., Review of Particle Physics, Phys. Lett. B 592 (2004) 1.

[5] T. Alexopoulos et al., Phys. Rev. D 70 (2004) 092006.

[6] A. Lai et al, Phys. Lett. B 602 (2004) 41.

[7] F. Ambrosino et al., Phys. Lett. B 632 (2006) 43.

[8] F. Ambrosino et al, Phys. Lett. B 626 (2005) 15.

[9] C. Amsler et al, Review of Particle Physics, Phys. Lett. B 667 (2008) 1.

[10] F. Ambrosino et al., Phys. Lett. B 636 92006) 173.

[11] F. Ambrosino et al., Eur. Phys. J. C 48 (2006) 767.

[12] J.R. Batley et al., Phys. Lett. B 653 (2007) 145.

[13] A. Lai et al., Phys. Lett. B 537 (2002) 28.

[14] A. Alavi-Harati et al., Phys. Rev. D 67 (2005) 012005.

[15] J.R. Batley at al., Eur. Phys. J. C 50 (2007) 329.

[16] F. Ambrosino et al., J. High Energy Phys. 02 (2008) 098.

[17] F. Ambrosino et al., Phys. Lett. B 666 (2008) 305.

[18] F. Ambrosino et al., Phys. Lett. B 632 (2006) 76. 
[19] F. Ambrosino et al., J. High Energy Phys. 01 (2008) 073.

[20] T. Alexopoulos et al., Phys. Rev. D 70 (2004) 092007.

[21] F. Ambrosino et al., Phys. Lett. B 636 (2006) 166.

[22] O.P. Yushchenko et al., Phys. Lett. B 589 (2004) 111.

[23] A. Lai et al., Phys. Lett. B 604 (2004) 1.

[24] F. Ambrosino et al., J. High Energy Phys. 12 (2007) 105.

[25] O.P. Yushchenko et al., Phys. Lett. B 581 (2004) 31.

[26] A. Lai et al., Phys. Lett. B 647 (2007) 341.

[27] A. Kastner and H. Neufeld, Eur. Phys. J. C 57 (2008) 541.

[28] V. Cirigliano, M. Gianotti, H. Neufeld, J. High Energy Phys. 11 (2008) 006.

[29] S. Descotes-Genon and B. Moussallam, Eur. Phys. J. C 42 (2005) 403.

[30] B. Ananthanarayan and B. Moussallam, J. High Energy Phys. 06 (2004) 047.

[31] W.J. Marciano, Phys. Rev. Lett. 93 (2004) 231803.

[32] P.A. Boyle et al., Phys. Rev. Lett. 100 (2008) 141601.

[33] E. Follana, C.T.H. Davies, G.P. Lepage, J. Shigemitsu, Phys. Rev. Lett. 100 (2008) 062002.

[34] J.C. Hardy, I.S.Towner, Phys. Rev. C 79 (2009) 055502.

[35] W.J. Marciano, A. Sirlin, Phys. Rev. Lett. 96 (2006) 032002.

[36] A. Czarnecki, W.J. Marciano, A. Sirlin, Phys. Rev. D 70 (2004) 093006.

[37] G. Isidori, P. Paradisi, Phys. Lett. B 639 (2006) 499. 